Provided for non-commercial research and education use. Not for reproduction, distribution or commercial use.

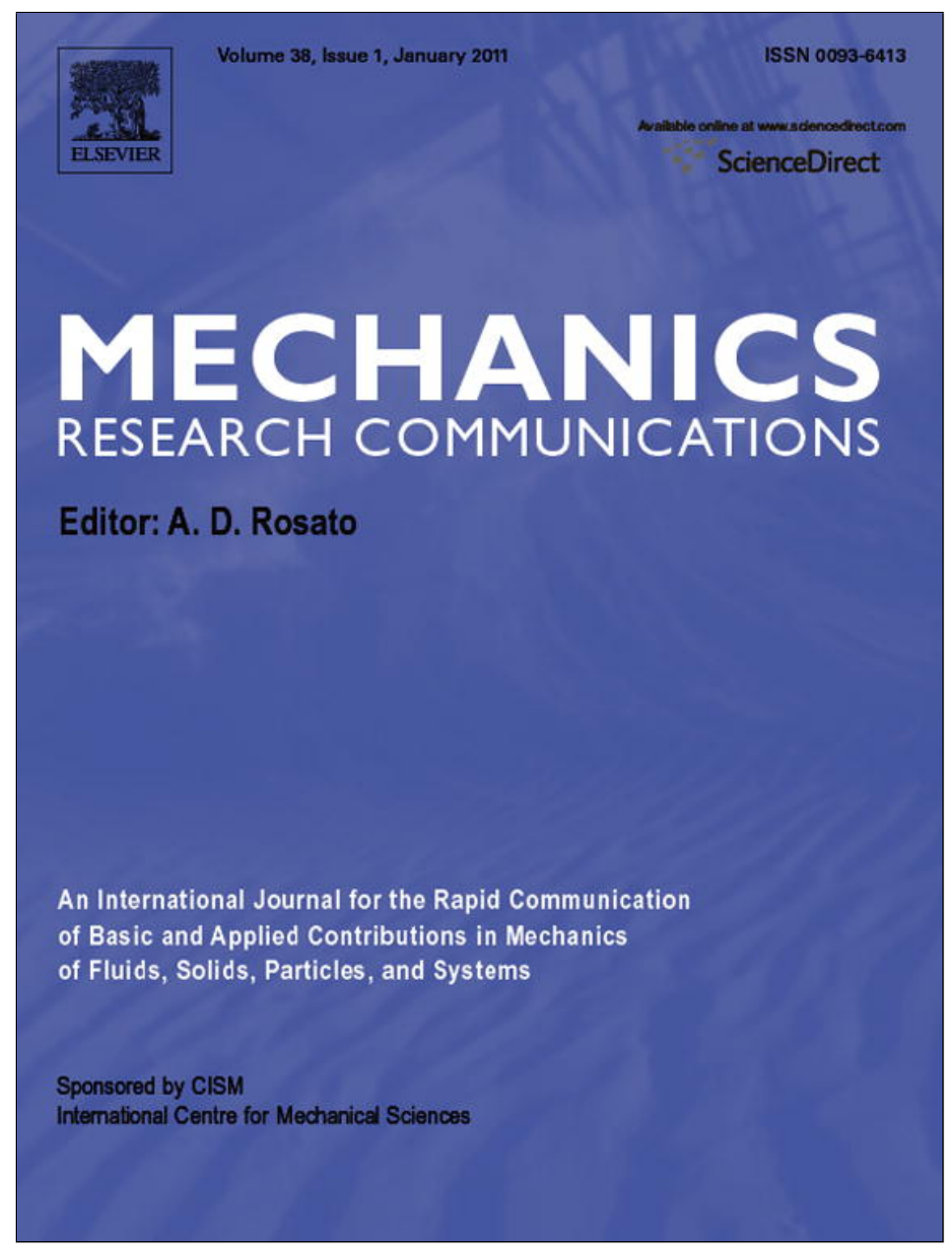

This article appeared in a journal published by Elsevier. The attached copy is furnished to the author for internal non-commercial research and education use, including for instruction at the authors institution and sharing with colleagues.

Other uses, including reproduction and distribution, or selling or licensing copies, or posting to personal, institutional or third party websites are prohibited.

In most cases authors are permitted to post their version of the article (e.g. in Word or Tex form) to their personal website or institutional repository. Authors requiring further information regarding Elsevier's archiving and manuscript policies are encouraged to visit:

http://www.elsevier.com/copyright 


\title{
Nonlocal vibration of carbon nanotubes with attached buckyballs at tip
}

\author{
T. Murmu*, S. Adhikari \\ College of Engineering, Swansea University, Singleton Park, Swansea SA2 8PP, Wales, UK
}

\section{A R T I C L E I N F O}

\section{Article history:}

Received 9 August 2010

Received in revised form

12 November 2010

Available online 30 November 2010

\section{Keywords:}

Carbon nanotubes

Buckyballs

Nonlocal elasticity

Frequency shift

\begin{abstract}
A B S T R A C T
Nonlocal longitudinal vibration of single-walled-carbon-nanotubes (SWCNTs) with attached buckyballs is considered. Attached buckyball at the tip of a SWCNT can significantly influence the resonance frequency of the vibrating system. Closed-form nonlocal transcendental equation for vibrating system with arbitrary mass ratio i.e. mass of buckyball to mass of SWCNT is derived. Nonlocal elasticity concept is employed to develop the frequency equations. Explicit analytical expressions of axial frequencies are proposed when mass of the attached buckyball is larger than the mass of SWCNT. Nonlocal longitudinal frequencies are validated with existing molecular dynamic simulation result. For arbitrary mass ratios, the frequency shifts in SWCNT due to (i) added buckyballs and (ii) nonlocal-effects are investigated. The present communication may be useful when designing tuneable resonator in NEMS applications.
\end{abstract}

(C) 2010 Elsevier Ltd. All rights reserved.

\section{Introduction}

Experimental (Ruud et al., 1994; Wong et al., 1997; Kasuya et al., 1997) and atomistic simulations (Chowdhury et al., 2010a) have evidenced a significant 'size-effect' in the mechanical properties when the dimensions of the nanostructures become 'small'. Size-effects are related to atoms and molecules that constitute the materials. Atomistic discrete methods, such as molecular mechanics simulation (Chowdhury et al., 2010b) are justified in the analysis of nanostructures. However the approach is computationally exorbitant for nanostructures with large numbers of atoms (Chowdhury et al., 2010a,b). Thus, research analyses generally have been carried out via classical continuum mechanics approach. Extensive research (Ru, 2001; Yoon et al., 2003; Behfar and Naghdabadi, 2005; Liew et al., 2006) over the past decade has shown that classical continuum theories are able to predict the performance of 'large' nanostructures reasonably well. The classical continuum theories include Euler-Bernoulli theory (Naguleswaran, 2006), Timoshenko beam theory (Timoshenko, 1953), Kirchhoff's plate theory (Reddy, 2007a), Mindlin plate theory (Mindlin, 1951) and classical shell theories (Jaunky and Knight, 1999). However classical continuum models are considered scale-free and it lacks the accountability of the effects arising from the small-scale. Thus, application of classical continuum models may be questionable in the analysis of nanostructures such as carbon nanotubes.

\footnotetext{
* Corresponding author. Tel.: +44 07742214778; fax: +44 1792295676 E-mail addresses: T.Murmu@swansea.ac.uk, murmutony@gmail.com (T. Murmu).
}

Recently there have been research efforts (Kong et al., 2008; Kahrobaiyan et al., 2010) to bring in the scale-effects and physics within the formulation by amending the traditional classical continuum mechanics. One widely promising size-dependant continuum theory is the nonlocal elasticity theory pioneered by Eringen (1983). In the nonlocal elasticity theory, the small-scale effects are captured by assuming that the stress at a point is a function of the strains at all points in the domain. Nonlocal theory considers long-range inter-atomic interaction and yields results dependent on the size of a body (Eringen, 1983). Some drawbacks of the classical continuum theory could efficiently be avoided and the size-dependent phenomena can be reasonably explained by nonlocal elasticity (Sudak, 2003; Lu, 2007; Reddy, 2007b; Heireche et al., 2008; Wang and Duan, 2008; Reddy and Pang, 2008; Li and Wang, 2009; Aydogdu, 2009a,b; Murmu and Pradhan, 2009; Lim and Yang, 2010).

Cylindrical fullerenes are known as carbon nanotubes, while spherical fullerenes (Fig. 1a) are referred to as buckyballs (Rao et al., 1995; Hermanson, 2008). As nanobuds (Zhao et al., 2010) can be obtained by adjoining fullerene to carbon nanotubes span (Fig. 1b), buckyballs can be added at the tip of carbon nanotubes for probable nanosensors and nanoresonator applications (Fig. 1c). Resonance based sensor offer significant potential of achieving the high-fidelity requirement of many sensing applications. The resonant frequency is sensitive to the resonator mass. The change in the attached mass on the resonator causes a shift to the resonant frequency. Attached buckyballs or added atoms at the tip of a SWCNT (Fig. 1c) can influence frequency change in vibrating systems (Park et al., 2005). Nanotubes have potential of being used as nanomechanical resonators in atomic-scale mass sensor. Likewise 
a

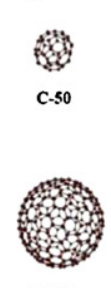

C-260
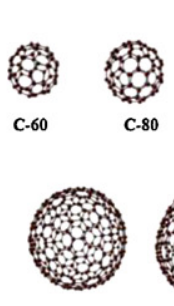

C-320
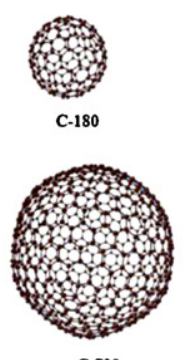

b

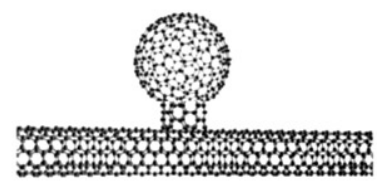

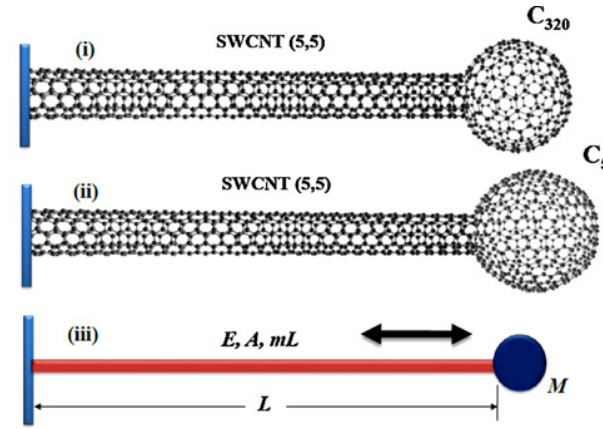

Fig. 1. (a) Standard carbon buckyballs. (b) Atomic configuration of attached buckyball in a nanobud configuration. (c) Atomic configuration of a SWCNT (5, 5) with attached buckyballs at the tip (i) $C_{320}$ (ii) $C_{500}$ (iii) mathematical idealization of a nanorod with a lumped mass.

carbon nanotubes with added buckyballs can be effectively used as tuneable nanoresonators in NEMS application.

On view of the above discussions, in this research communication, we examine the nonlocal-effects in the longitudinal dynamic properties of single-walled-carbon-nanotubes (SWCNTs) with attached buckyballs. For the abovementioned problems we derive the governing equations for longitudinal vibration based on nonlocal elasticity theory (Eringen, 1983). Nonlocal axial frequencies are validated with existing molecular dynamic simulations studies. Analytical expressions of nonlocal frequencies are derived when the mass of the attached buckyball is larger than the mass of SWCNT. Closed-form nonlocal transcendental equation for vibrating system with arbitrary mass ratio i.e. mass of buckyballs to mass of SWCNT is derived. The frequency shifts due to (i) added buckyballs and (ii) nonlocal-effects are considered for arbitrary mass ratios. The following results and discussion of this study can be utilized for the design of a tuneable nanoresonator.

\section{Nonlocal elasticity}

According to the nonlocal elasticity, the basic partial-integral stress relation for an isotropic, linear and homogenous nonlocal elastic body neglecting the body force is expressed as (Eringen, 1983)

$\sigma_{i j}(x)=\int_{V} \phi\left(\left|\mathbf{x}-\mathbf{x}^{\prime}\right|, \alpha\right) t_{i j} d \mathbf{V}\left(\mathbf{x}^{\prime}\right), \quad \forall x \in \mathbf{V}$

The terms $\sigma_{i j}$ and $t_{i j}$ are the nonlocal stress and classical stress tensors, respectively. The volume integral is over the region $\mathbf{V}$ occupied by the body. The kernel function $\phi\left(\left|\mathbf{x}-\mathbf{x}^{\prime}\right|, \alpha\right)$ is the nonlocal modulus. The nonlocal modulus acts as an attenuation function incorporating into constitutive equations the nonlocal effects at the reference point $\mathbf{x}$ produced by local strain at the source $\mathbf{x}^{\prime}$. The term $\left|\mathbf{x}-\mathbf{x}^{\prime}\right|$ represents the distance in the Euclidean form and $\alpha$ is a material constant that depends on the internal, $a$, (e.g. lattice parameter, granular size, distance between the $\mathrm{C}-\mathrm{C}$ bonds) and external characteristics lengths, $\ell$, (e.g. crack length, wave length). Material constant $\alpha$ is defined as $\alpha=e_{0} a / \ell$. Here $e_{0}$ is a constant for calibrating the model with experimental results and other validated models. The parameter $e_{0}$ is estimated such that the relations of the nonlocal elasticity model could provide satisfactory approximation to the atomic dispersion curves of the plane waves with those obtained from the atomistic lattice dynamics (Eringen, 1983). Term $e_{0}$ is closely related to complex internal microstructures of materials and thus no specific values have been determined till now (Li and Wang, 2009).

The nonlocal partial-integral equation couples the stress due to nonlocal elasticity and the stress due to classical elasticity. The partial-integral equation is generally difficult to solve analytically. Thus a differential form of nonlocal elasticity equation is often used. According to Eringen (1983), the following expression of nonlocal modulus can be used as

$\phi(|\mathbf{x}|, \alpha)=\left(2 \pi \ell^{2} \alpha^{2}\right)^{-1} K_{0}\left(\frac{\sqrt{\mathbf{x} \cdot \mathbf{x}}}{\ell \alpha}\right)$

where $K_{0}$ is the modified Bessel function. Assuming the kernel function $\phi$ as the Green's function, Eringen (1983) proposed a differential form of the nonlocal constitutive relation as

$\left(1-\alpha^{2} \ell^{2} \nabla^{2}\right) \sigma_{i j}=t_{i j}$

where $\nabla^{2}$ is the Laplacian. The nonlocal differential equation (Eq. (3)) couples the stress due to nonlocal elasticity and the stress due to classical elasticity. When $\alpha=0$ both classical and nonlocal stresses are same.

\section{Equation of motion}

\subsection{Nonlocal rod theory}

The nonlocal constitutive relation (Eq. (3)) in one-dimensional form reduces to (Reddy, 2007b)

$\sigma(x)-\left(e_{0} a\right)^{2} \sigma^{\prime \prime}(x)=E \varepsilon(x)$

where $E$ and $\varepsilon$ denote the Young's modulus and the strain in SWCNT, respectively. Term $x$ denotes the longitudinal coordinate. Term $\left(e_{0} a\right)$ is the dimensional nonlocal parameter.

We use the relation for stress resultant as

$N=\int_{A} \sigma(x) d A$

where $A$ is the cross-section of the uniform SWCNT.

Using Eqs. (4) and (5) we get the constitutive relation as

$N(x)-\left(e_{0} a\right)^{2} N^{\prime \prime}(x)=E A u^{\prime}(x, t)$

where' denotes the $(\partial / \partial x)$ and " denotes the $\left(\partial^{2} / \partial x^{2}\right)$.

We use the Euler-Lagrange equation in the domain $0<x<L$ as

$N^{\prime}(x)+f(x, t)=m \ddot{u}(x, t)$

where. denotes the $\left(\partial^{2} / \partial t^{2}\right)$. Here $m$ and $u(x, t)$ are the mass per unit length and axial displacements of the SWCNT; and $f(x, t)$ is the axially distributed force.

Using Euler-Lagrange expression, the nonlocal governing equation can be expressed as (Reddy, 2007a; Aydogdu, 2009a,b; Filiz and Aydogdu, 2010)

$-E A u^{\prime \prime}(x, t)-f(x, t)-\left(e_{0} a\right)^{2} f^{\prime \prime}(x, t)+m \ddot{u}(x, t)-\left(e_{0} a\right)^{2} m \ddot{u}{ }^{\prime \prime}(x, t)=0$ 


\subsection{Longitudinally vibrating nanotube-buckyball systems}

Consider a single-walled carbon nanotube (SWCNT) with attached buckyball (Fig. 1c). The SWCNT is assumed to be of length $L$. The mathematical idealization of the vibrating system is shown in Fig. 1c(iii). The SWCNT is assumed as a nonlocal rod. The SWCNT is considered to be slender and modelled by nonlocal rod theory.

Consider the solution of Eq. (8) as

$u(x, t)=U(x) e^{i \omega t}$

where $\omega$ is the natural frequency and $i=\sqrt{-1}$. Considering normalizing coordinate, $X=x / L$ and using Eq. (9) Euler-Lagrange equation (neglecting $f(x, t)$ ) can be transformed to space variables form as

$U^{\prime \prime}(X)+\lambda^{2} U(x)=0$,

where

$\lambda^{2}=\frac{\Omega^{2}}{\left[1-\left(e_{0} a / L\right)^{2} \Omega^{2}\right]}$

and

$\Omega^{2}=\frac{m \omega^{2} L^{2}}{E A}$

Term $U(X)$ can be expressed as

$U(X)=A \cos \lambda X+B \sin \lambda X$

where $A$ and $B$ are determined from boundary conditions.

Suppose there is an attached buckyballs at the tip of SWCNT (Fig. 1c). The boundary conditions for single-walled carbon nanotubes with attached buckyballs can be expressed as

$(u)_{X=0}=0$

and

$\left(\frac{E A}{L}\right)\left(\frac{\partial u}{\partial X}\right)_{X=1}+\left(e_{0} a\right)^{2}\left(\frac{m}{L}\right)\left(\frac{\partial^{3} u}{\partial X \partial t^{2}}\right)_{X=1}=-M_{\text {BUCKYBALL }}\left(\frac{\partial^{2} u}{\partial t^{2}}\right)_{X=1}$

Here the term $M_{\text {BUCKYBALL }}$ denotes the mass of the carbon buckyball. It should be noted that boundary condition represented by Eq. (15) is the nonlocal boundary condition and not the classical one.

Applying the boundary conditions (Eqs. (14) and (15)), we get the governing nonlocal transcendental frequency equation as

$\frac{1}{\Delta M}=\lambda \tan \lambda$

where the term $\lambda$ is defined as Eq. (11) and $\Delta M$ is the ratio of mass of the buckyballs to the mass of SWCNT as

$\Delta M=\frac{M_{\mathrm{BUCKYBALLS}}}{M_{\mathrm{CNT}}}$

When the SWCNT is solo and there are no buckyballs we have $\Delta M=0$. Thus Eigen value equation $(\cos \lambda=\Delta M \lambda \sin \lambda)$ reduces to conventional frequency equation: $\cos \lambda=0$ (Aydogdu, 2009a,b; Filiz and Aydogdu, 2010).

\subsection{Frequency relation for nanotube-buckyball system when $M_{C N T}<M_{B U C K Y B A L L S}$}

When the mass of SWCNT is small compared to the mass of buckyballs, then $1 / \Delta M \rightarrow 0$ and $\lambda \rightarrow 0$. This implies that $\tan \lambda \rightarrow \lambda$; and we can assume that $\tan \lambda=\lambda$. Thus we obtain a simple relation for nonlocal resonant frequency and mass ratio as

$\lambda^{2}=\left(\frac{1}{\Delta M}\right)$

where $\lambda$, which is a function of nonlocal parameter, is defined in Eq. (11).
Using Eq. (11), explicit nonlocal frequency as a function of mass ratio $\Delta M$ and nonlocal parameter is evaluated as

$\Omega_{R}=\sqrt{\left(\frac{1}{\Delta M} /\left[1+\left(\frac{e_{0} a}{L}\right)^{2}\left(\frac{1}{\Delta M}\right)\right]\right)}$

A better assumption of Eq. (19) can be found by considering the second term of $\tan \lambda$ in Taylor series, i.e.

$\tan \lambda=\lambda+\frac{\lambda^{3}}{3}$

Using Eq. (18), we can have explicit relation for nonlocal frequency as

$\Omega_{R}=\sqrt{\left(\frac{1}{\Delta M} /\left[1+\frac{1}{3}\left(\frac{1}{\Delta M}\right)+\left(\frac{e_{0} a}{L}\right)^{2}\left(\frac{1}{\Delta M}\right)\right]\right)}$

When the nonlocal parameter, $e_{0} a=0$, the frequency of vibrating system reduces to the longitudinal classical frequency (Timoshenko, 1937). This coincides with the well-known relation by Rayleigh method (Rayleigh, 1954). This new expression (Eq. (21)) can be viewed as Rayleigh-nonlocal frequency relation for longitudinal vibration.

Similarly considering the first three terms of $\tan \lambda$ in Taylor series, i.e. $\tan \lambda=\lambda+\lambda^{3} / 3+2 \lambda^{5} / 15$, we have the nonlocal frequency relation as

$\Omega_{R}=\sqrt{\left(\frac{1}{\Delta M} /\left[1+\frac{1}{3}\left(\frac{1}{\Delta M}\right)+\frac{2}{15}\left(\frac{1}{\Delta M}\right)^{2}+\left(\frac{e_{0} a}{L}\right)^{2}\left(\frac{1}{\Delta M}\right)\right]\right)}$

The behaviour of these frequency relations with different mass ratios is discussed in the next section.

\section{Results and discussion}

\subsection{Nonlocal longitudinal frequencies vs. molecular dynamics simulation}

To validate the frequencies based on present continuum nonlocal rod theory, an armchair SWCNT $(5,5)$ without attached buckyball is considered. Nonlocal fundamental axial frequencies $(f=\omega / 2 \pi)$ are computed and compared with molecular dynamics (MD) simulation result (Cao et al., 2006). The geometric and material properties of SWCNT are length, $L=24.4 \mathrm{~nm}$, density $\rho=9.517 \times 10^{3} \mathrm{~kg} / \mathrm{m}^{3}$, thickness, $t=0.08 \mathrm{~nm}$. The frequencies by local elasticity, nonlocal elasticity and MD simulation are listed in Table 1. Good agreement between the analytical results and MD simulation is observed. However the frequencies obtained by local elasticity are slightly over-predicted compared to the frequencies by MD. This can be accounted as: the small scale-effects are included in MD simulation (Chowdhury et al., 2010a, b) while classical model (Timoshenko, 1937) is independent of the small scale-effects. The gap between the frequencies by local elasticity and MD simulation are diminished by applying nonlocal effects $\left(e_{0} a \neq 0\right)$.

The frequencies of vibrating systems are however sensitive to the choice of nonlocal parameters. Proper choice of nonlocal parameter for a particular system is thus important. From Table 1, we observe that $e_{0} a=1 \mathrm{~nm}$ gives the best agreement with the MD simulation. This value of nonlocal parameter can be considered as the "best value" of nonlocal parameter for the present vibrating system; and can be used for future applications of the model. However it should be noted that the choice of nonlocal parameters need to be optimized for each problem geometry and boundary conditions. Nonlocal based analysis can be used for nanostructures provided the dependence of the small-scale effect on dimensional scaling is properly accounted for. 
Table 1

Comparison of longitudinal natural resonance frequencies $(f=\omega / 2 \pi)$ of armchair single-walled carbon nanotubes $(5,5)$ with existing molecular dynamics simulation result.

\begin{tabular}{|c|c|c|c|c|c|c|}
\hline \multirow[t]{2}{*}{ Nonlocal parameter $\left(e_{0} a\right)$} & \multirow[t]{2}{*}{ Classical model (local) } & \multicolumn{4}{|c|}{ Scale dependent nonlocal model } & \multirow{2}{*}{$\begin{array}{l}\text { Molecular dynamics } \\
\text { simulation (Cao et al., } 2006\end{array}$} \\
\hline & & $\left(e_{0} a=0.5 \mathrm{~nm}\right)$ & $\left(e_{0} a=1.0 \mathrm{~nm}\right)$ & $\left(e_{0} a=1.5 \mathrm{~nm}\right)$ & $\left(e_{0} a=2.0 \mathrm{~nm}\right)$ & \\
\hline Natural frequencies $(f)$ in $\mathrm{Hz}$ & $5.50 \times 10^{11}$ & $5.49 \times 10^{11}$ & $5.45 \times 10^{11}$ & $5.40 \times 10^{11}$ & $5.32 \times 10^{11}$ & $5.44 \times 10^{11}$ \\
\hline
\end{tabular}

4.2. Nonlocal effects in longitudinal frequencies when

$M_{C N T}<M_{B U C K Y B A L L}$

Next, we examine the variation of nonlocal frequency against the mass ratio of the vibrating system. We assume that the mass of SWCNT is less than the mass of buckyballs (Fig. 2a). Small range values i.e. $0<(1 / \Delta M)<0.5$ is considered for the present study. The plot of first mode frequency parameter $\Omega$ with $(1 / \Delta M)$ is shown in Fig. 2b. As the values of $(1 / \Delta M)$ increases, the frequency parameter $\Omega$ increase. The increase of $\Omega$ is due to the increasing axial rigidity of the vibrating system (Timoshenko, 1937). Applying increasing value of the nonlocal parameter, $\left(e_{0} a / L\right)$ the frequency parameter $\Omega$ decrease for the entire mass ratio $(1 / \Delta M)$ considered. This reduction in frequency parameter is due to the incorporation of small-scale effects (Reddy, 2007b; Aydogdu, 2009a,b; Filiz and Aydogdu, 2010).

Plots with three different assumptions of Taylor series are also shown in this study. The three assumptions of nonlocal frequency are based on Eqs. (19), (21) and (22), respectively. Curves for local elasticity theory with all the three assumptions are acceptable till $(1 / \Delta M)=0.1$. Beyond $(1 / \Delta M)=0.1$ the assumptions would deliver different values of frequency parameter. However, interesting to note, with increasing $\left(e_{0} a / L\right)$, all three assumptions hold good for frequency solutions till $(1 / \Delta M)=0.5$.

For all assumptions considered employing local or nonlocal elasticity, the Rayleigh-nonlocal frequency relation $\Omega_{R}=$ $\sqrt{(1 / \Delta M) /\left[1+1 / 3(1 / \Delta M)+\left(e_{0} a / L\right)^{2}(1 / \Delta M)\right]}$ yields the more accurate nonlocal axial frequencies for range of $0<(1 / \Delta M)<0.5$. This is because when using the approximation Eq. (18) with more numbers of terms in Taylor series, it induces error in the frequency values. Thus Eq. (21) would be the better expression of nonlocal frequency.

\subsection{Nonlocal effects in longitudinal frequencies with arbitrary mass ratios}

\subsubsection{Frequency shift in SWCNT due to added buckyballs}

Next, arbitrary mass ratios of the vibrating system are considered. For any mass ratio $(1 / \Delta M)$, the transcendental equation (Eq.
(16)) is computed numerically (Forsythe et al., 1976). We introduce a term 'frequency shift percent' (FSP) for the present study.

Resonance frequency shift percent is defined as

FSP $=100 \times \frac{\Omega_{\text {NO BUCKYBALL }}-\Omega_{\text {WITH BUCKYBALL }}}{\Omega_{\text {NO BUCKYBALL }}}$

The term FSP reflects the deviation of frequency values of the vibrating system from the SWCNT with no added buckyballs. The resonant frequency is sensitive to the resonator mass. The change in the attached mass (buckyballs) on the resonator causes a shift to the resonant frequency (Adhikari and Chowdury, 2010). Further, Chiu et al. (2008) utilized this detection of shifts in the resonance frequency of the nanotubes to quantify the nanotubes resonator vibration characteristics.

Frequency shift percent (FSP) is plotted for various mass ratios $1 / \Delta M$ and is shown in Fig. 3a. When $1 / \Delta M \rightarrow 0,\left(M_{\text {BUCKYBALL }} \rightarrow \infty\right)$ FSP is $100 \%$. Frequency shift of the vibrating system increases with increasing the attached nano-mass with respect to the mass of SWCNT. Thus attaching different buckyballs at the tip, the resonance frequency can be tuned accordingly and can be used as tuneable nanoresonator.

As the mass of SWCNT increase, the FSP decreases. This decreasing trend (Fig. 3a) is much influenced (reduced) by increasing dimensionless nonlocal parameter $\left(e_{0} a / L\right)$. This trend can be justified by experimental studies (Ruud et al., 1994; Wong et al., 1997; Kasuya et al., 1997; Juhasz et al., 2004).

FSP will quantify the change in the resonant frequency due to the added buckyballs or biomolecules. The parameter FSP will thus help in design of vibrating carbon nanotubes based sensor or resonators in NEMS application.

\subsubsection{Frequency shift in vibrating system due to nonlocal-effects}

Based on nonlocal elasticity theory, we have derived the closed form transcendental frequency equation to analyze the effects of nonlocal parameter. To see the nonlocal effect on vibrating SWCNTattached-buckyballs, nonlocal frequency shift percent (NFSP) is computed for SWCNT attached with various standard buckyballs (Fig. 3b). NFSP represents the shift in frequency in vibrating nanostructures when the nonlocal effects are ignored.
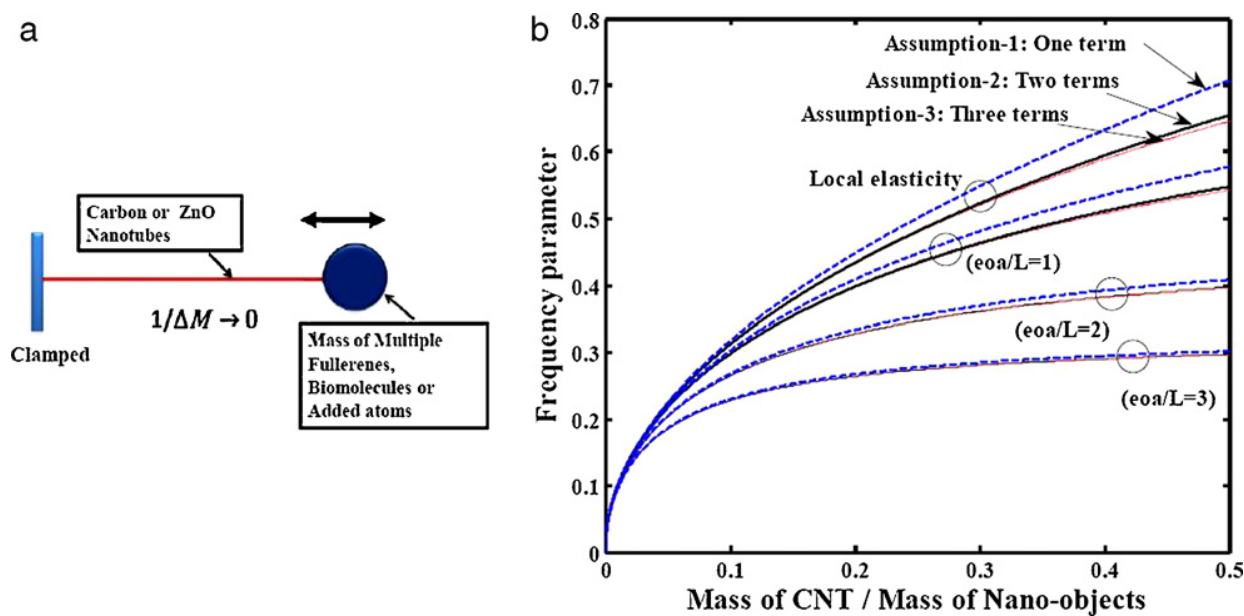

Fig. 2. (a) Mathematical idealization when mass of attached nano-object (viz. buckyballs, biomolecules and added atoms) is large compared to SWCNT. (b) Variation of frequency parameter with mass ratio for different dimensionless nonlocal parameter for smaller (1/ $\Delta M)$ range: $0-0.5$. 

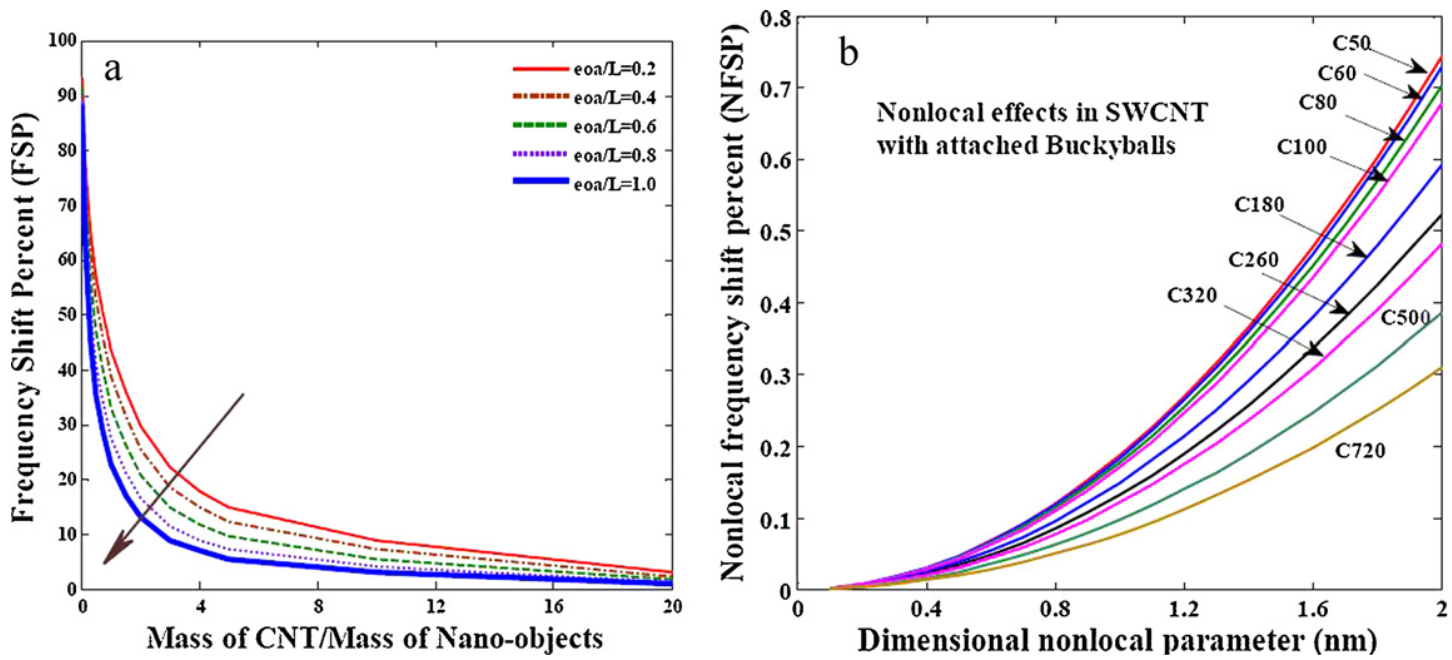

Fig. 3. (a) Effect of mass ratio on frequency shift percent (FSP) of the vibrating system for various dimensionless non-local parameters. (b) Effect of nonlocal parameter on nonlocal frequency shift percent (NFSP) parameter for vibrating system with different standard carbon buckyballs.

Table 2

Ratio $(\Delta M)$ of SWCNT $(5,5)$ and buckyballs (spherical fullerenes).

\begin{tabular}{lllllllll}
\hline Buckyballs & $C_{50}$ & $C_{60}$ & $C_{80}$ & $C_{100}$ & $C_{180}$ & $C_{260}$ & $C_{320}$ \\
\hline$\Delta M$ & 0.0504 & 0.0605 & 0.0806 & 0.1008 & 0.1814 & 0.2620 & 0.3225 & 0.5039 \\
\hline
\end{tabular}

Nonlocal frequency shift percent is defined as

$\mathrm{NFSP}=100 \times \frac{\Omega_{\text {Local }}-\Omega_{\text {Nonlocal }}}{\Omega_{\text {Local }}}$

Vibrating systems are assumed by attaching different buckyballs at the tip of SWCNT. A SWCNT $(5,5)$ for length $L=24.4 \mathrm{~nm}$ is considered. The radius of SWCNT is determined as $R=$ $0.246 / 2 \pi \sqrt{n^{2}+n m+m^{2}}$. The mass of SWCNT considered as $M_{\mathrm{CNT}}=\rho A L=1.9789 \times 10^{-23} \mathrm{~kg}$. Different standard carbon buckyballs considered are $C_{50}, C_{60}, C_{80}, C_{100}, C_{260}, C_{320}, C_{500}$ and $C_{720}$. The practical mass ratios $\Delta M$ of the vibrating system with different standard buckyballs are listed in Table 2.

Nonlocal frequency shift percent (NFSP) is plotted against dimensional nonlocal parameter for SWCNT attached with various standard buckyballs (Fig. 3b). Dimensional nonlocal parameter, $e_{0} a=0-2.0 \mathrm{~nm}$ (Wang and Duan, 2008; Aydogdu, 2009a,b) is employed. One can see that increasing the dimensional nonlocal parameter increases the nonlocal frequency shift. Based on the nonlocal elasticity theory, long-range interactions are taken account in the analysis that makes the vibrating system stiffer. This trend is obvious when the attached buckyballs is small compared with that of the SWCNT. The figure shows that with lighter buckyballs (viz. $C_{50}, C_{60}, C_{80}$ ) the nonlocal effects on NFSP are more pronounced compared to SWCNT with heavier buckyballs (viz. $C_{320}, C_{500}, C_{720}$ ).

Carbon nanotube based nanostructures has the potential of being used as nanomechanical resonators. Single-walled or multiwall carbon nanotubes with added buckyballs can be effectively used as tuneable nanoresonators in NEMS applications. As shown in this paper, using different buckyballs the frequency of the combined resonator can be changed according to the requirements. For the proper design of such nanocomponents in NEMS one need to consider nonlocal effects and atomic forces to obtain solutions with acceptable accuracy. Neglecting these effects in some cases may result in inaccurate solutions and hence incorrect designs. The present analysis includes nonlocal effects with acceptable accuracy in the solution and thus may be useful when designing tuneable resonator in NEMS applications.

\section{Conclusions}

This research communication focuses on a phenomenological approach to highlight the nonlocal-effects in longitudinal dynamic properties of SWCNT with added buckyballs. The SWCNT is assumed to be nonlocal rod while the buckyballs are assumed to be as nonlocal point mass. Explicit expressions relating nonlocal frequencies and mass ratio are developed when the mass of the attached buckyball is larger than the mass of SWCNT. A closedform nonlocal transcendental equation is derived for arbitrary mass ratio. Axial resonant frequency of the vibrating system is sensitive to the attached mass. With increase of the mass of buckyballs in vibrating system, the shift in the frequencies increases. Also the shift in the nonlocal frequencies increases with the increasing nonlocal parameter; and is more pronounced for lighter buckyballs. The terms frequency shift percentage (FSP) and nonlocal frequency shift percentage (NFSP) could be important for nanoresonators. These terms will quantify the change in the resonant frequency due to the added buckyballs and correct incorporation of nonlocal effects. The parameter FSP and NFSP will thus help in design of vibrating carbon nanotubes based tuneable resonators in NEMS application. In summary, this nonlocal study may be employed for the design of a tuneable resonator with proper selection of nonlocal parameter.

\section{References}

Adhikari, S., Chowdury, R., 2010. The calibration of carbon nanotube based bionanosensors. J. Appl. Phys. 107, 124322.

Aydogdu, M., 2009a. A general nonlocal beam theory: its application to nanobeam bending, buckling and vibration. Physica E 41, 1651-1655.

Aydogdu, M., 2009b. Axial vibration of the nanorods with the nonlocal continuum rod model. Physica E 41, 861-864.

Behfar, K., Naghdabadi, R., 2005. Nanoscale vibrational analysis of a multi-layered graphene sheet embedded in an elastic medium. Compos Sci. Technol. 65, 1159-1164.

Cao, G., Chena, X., Kysarb, J.W., 2006. Thermal vibration and apparent thermal contraction of single-walled carbon nanotubes. J. Mech. Phys. Solids 54, 1206-1236.

Chiu, H.Y., Hung, P., Postma, H.W.C., Bockrath, M., 2008. Atomic-scale mass sensing using carbon nanotube resonators. Nano Lett. 8, 4342-4346.

Chowdhury, R., Adhikari, S., Wang, C.W., Scarpa, F., 2010a. A molecular mechanics approach for the vibration of single walled carbon nanotubes. Comput. Mater. Sci. $48,730-735$. 
Chowdhury, R., Adhikari, S., Scarpa, F., 2010b. Vibrational analysis of ZnO nanotubes: a molecular mechanics approach. Appl. Phys. A, doi:10.1007/s00339-010-5995$3<$.

Eringen, A.C., 1983. On differential equations of nonlocal elasticity and solutions of screw dislocation and surface waves. J. Appl. Phys. 54, 4703-4710.

Filiz, S., Aydogdu, M., 2010. Axial vibration of carbon nanotube heterojunctions using nonlocal elasticity. Comput. Mater. Sci. 49, 619-627.

Forsythe, G.E., Malcolm, M.A., Moler, C.B., 1976. Computer Methods for Mathematical Computations. Prentice-Hall.

Heireche, H., Tounsi, A., Benzair, A., Mechab, I., 2008. Sound wave propagation in single-walled carbon nanotubes with initial axial stress. J. Appl. Phys. 104, 014301.

Hermanson, G.T., 2008. Buckyballs, Fullerenes, and Carbon Nanotubes, 2nd ed. Bioconjugate Techniques, pp. 627-648.

Jaunky, N., Knight, 1999. An assessment of shell theories for buckling of circular cylindrical laminated composite panels loaded in axial compression. Int. J. Solids Struct. 36 (25), 3799-3820.

Juhasz, J.A., Best, S.M., Brooks, R., Kawashita, M., Miyata, N., Kokubo, T., Nakamura, T., Bonfield, W., 2004. Mechanical properties of glass-ceramic A-W-polyethylene composites: effect of filler content and particle size. Biomaterials 25, 949-955.

Kahrobaiyan, M.H., Asghari, M., Rahaeifard, M., Ahmadian, M.T., 2010. Investigation of the size-dependent dynamic characteristics of atomic force microscope microcantilevers based on the modified couple stress theory. Int. J. Eng. Sci. 48, 1985-1994.

Kasuya, A., Sasaki, Y., Saito, Y., Tohji, K., Nishina, Y., 1997. Evidence for sizedependent discrete dispersions in single-wall nanotubes. Phys. Rev. Lett. 78, 4434-4437.

Kong, S.L., Zhou, S.J., Nie, Z.F., Wang, K., 2008. The size-dependent natural frequency of Bernoulli-Euler micro-beams. Int. J. Eng. Sci. 46, 427-437.

Li, X.F., Wang, B.L., 2009. Vibrational modes of Timoshenko beams at small scales. Appl. Phys. Lett. 94, 101903

Liew, K.M., He, X.Q., Kitipornchai, S., 2006. Predicting nanovibration of multi-layered graphene sheets embedded in an elastic matrix. Acta Mater. 54 (16), 4229-4236.

Lim, C.W., Yang, Y., 2010. New predictions of size-dependent nanoscale based on nonlocal elasticity for wave propagation in carbon nanotubes. J. Comput. Theor. Nanosci. 7, 988-995.

Lord Rayleigh, 1954. The Theory of Sound, 2nd ed. Dover, New York, 1.
Lu, P., 2007. Dynamic analysis of axially prestressed micro/nanobeam structures based on nonlocal beam theory. J. Appl. Phys. 101, 073504.

Mindlin, R.D., 1951. Influence of rotatory inertia and shear on flexural motions of isotropic elastic plates. J. Appl. Mech., ASME 18, 31-38.

Murmu, T., Pradhan, S.C., 2009. Buckling of biaxially compressed orthotropic plates at small scales. Mech. Res. Commun. 36, 933-938.

Naguleswaran, S., 2006. Vibration of an Euler-Bernoulli uniform beam carrying a rigid body at each end. Int. J. Mech. Eng. Ed. 34 (3), 194-210.

Park, S.H., Kim, J.S., Park, J.H., Lee, J.S., Choi, Y.K., Kwon, O.M., 2005. Molecular dynamics study on size-dependent elastic properties of silicon nanocantilevers. Thin Solid Films 492, 285-289.

Rao, C.N.R., Seshadri, R., Govindaraj, A., Sen, R., 1995. Fullerenes, nanotubes, onions and related carbon structures. Mater. Sci. Eng. R 15 (6), 209-262.

Reddy, J.N., 2007a. Nonlocal theories for bending, buckling and vibrations of beams. Int. J. Eng. Sci. 45, 288-307.

Reddy, J.N., 2007b. Theory and Analysis of Elastic Plates and Shells. CRC Press, Taylor and Francis.

Reddy, J.N., Pang, S.D., 2008. Nonlocal continuum theories of beams for the analysis of carbon nanotubes. J. Appl. Phys. 103, 023511.

Ru, C.Q., 2001. Axially compressed buckling of a doublewalled carbon nanotube embedded in an elastic medium. J. Mech. Phys. Solids 49, 1265-1279.

Ruud, J.A., Jervis, T.R., Spaepan, F., 1994. Nanoindention of Ag/Ni multilayered thin films. J. Appl. Phys. 75, 4969.

Sudak, L.J., 2003. Column buckling of multiwalled carbon nanotubes using nonlocal continuum mechanics. J. Appl. Phys. 94, 7281-7287.

Timoshenko, S., 1937. Vib. Prob. Eng., 2nd ed., New York.

Timoshenko, S., 1953. History of Strength of Materials. McGraw-Hill, New York.

Wang, C.M., Duan, W.H., 2008. Free vibration of nanorings/arches based on nonlocal elasticity. J. Appl. Phys. 104, 014303.

Wong, E.W., Sheehan, P.E., Lieber, C.M., 1997. Nanobeam mechanics: elasticity, strength, and toughness of nanorods and nanotubes. Science $277,1971-1975$.

Yoon, J., Ru, C.Q., Mioduchowski, A., 2003. Vibration of an embedded multiwall carbon nanotube. Comput. Sci. Technol. 63 (11), 1533-1542.

Zhao, P., Wang, P.J., Zhang, Z., Ren, M.J., Liu, D.S., 2010. First-principles study of the electronic transport properties of the carbon nanobuds. Physica B: Condens. Matter. 405 (8), 2097-2101. 